\title{
Effect of asymmetries of stiffness and mass on parametric instabilities of hollow rotor system \\ DOI: 10.36909/jer.ICCEMME.15745
}

\author{
Sandeep Chauhan*, Chandan Kumar**, Ashish Gupta***, Vikas Rastogi**** \\ *Assistant Professor, Department of Mechanical Engineering, G L Bajaj Institute of \\ Technology and Management, Greater Noida-201306, India \\ *Research Scholar, Dr. A.P.J. Abdul Kalam Technical University, Uttar Pradesh, Lucknow, \\ India \\ **Associate Professor, Department of Mechanical Engineering, BIT Sindari, 828123, India \\ ***Associate Professor, Department of Mechanical Engineering, G L Bajaj Institute of \\ Technology and Management, Greater Noida-201306, India \\ **** Professor, Design Engineering Centre, Department of Mechanical Engineering, Delhi \\ Technological University, Delhi-110042, India. \\ *Email: ashish.j001@gmail.com; Corresponding Author.
}

\begin{abstract}
The present work aims to understand the influence of the asymmetries on the dynamics of the hollow rotor system. The study comprises the analytical study through the extended Lagrangian Hamiltonian approach and validation conducted with the simulation study through the bond graphs. This study involves an investigation of the dynamics of the hollow rotor system through two case studies. First case study considers a hollow rotor system with small asymmetry in stiffness while another study considers a hollow rotor system with asymmetry in mass. The lumped analysis involves the development of a mathematical model by considering the symmetry breaking of a hollow rotor caused by mass or stiffness. The study shows good agreement in simulation and analytical results.
\end{abstract}

Key words: hollow rotor, asymmetric rotor, extended Lagrangian Hamiltonian mechanics, umbra time. 


\section{INTRODUCTION}

Most of the rotary machines used for engineering applications are asymmetric due to misalignment or unbalance. Sometimes, symmetric systems become asymmetric during service due to initiation and growth of fatigue crack, slit, notches, wear and tear, etc. The presence of a crack causes variations in stiffness, mass, and damping. These variations make the system asymmetric and alter the dynamic behavior of the rotor. Analysis of such a system is necessary for the accurate prediction of the presence of these asymmetries, which are dangerous for the structural health of the rotor system. The cross-sectional geometry also affects the dynamics of the rotor due to alteration in the stiffness of the rotor system. Mostly solid cylindrical shaped rotor used due to ease in construction and analysis. However, the attention of researchers shifts towards a hollow rotor-shaft system owing to a lesser weight of shafts for transmitting the same power and consequently lesser requirement of energy for operation. Asymmetries greatly affected the performance of the hollow rotor system due to lesser stiffness. Thus, it becomes necessary to analyze the effects of asymmetries on the dynamics of the hollow rotor.

Dimarogonas (1996) presented a paper to elaborate on several aspects of local flexibility associated with the asymmetries in a rotating system. While Kumar et al. (2009) elaborated various methodologies employed by researchers to understand the effect of asymmetries due to crack on the dynamics of a rotor system. Generally, the Lagrangian-based approach is preferred for analyzing dynamics of the rotating system due to its inherent characteristics of describing conservation laws associated with symmetry properties. Some studies have been initiated to enlarge the applicability of the Lagrange equation to a rotating system subjected to holonomic constraints and dissipative forces. Vujanovic and Djukic $(1978,1989,1973,1975)$ attempted to show the connection between symmetries and associated conservation law. Vujanovic $(1978,1989)$ proposed a new approach to obtain 
constants of motion of a rotary system with non-potential forces with non-holonomic constraints.

As any rotating component with asymmetries consists of non-potential and dissipative forces and in such a situation, Lagrangian-based approaches failed to explain satisfactorily dynamics of an asymmetric rotary system involving non-holonomic constraints, frictional forces, and time fluctuating parameters. Analysis of these systems requires knowledge of invariants of motion of the system due to the existence of a relation between symmetries and invariant of motions $(1977,1997)$ of such systems. Noether's theorem (1971) complemented the knowledge and contributed significantly to determine invariance properties. Extended Noether theorem together with Hamiltonian mechanics was found useful in understanding the dynamic behavior of such an asymmetric rotor system. For improving understanding in this direction, Mukherjee (1994) introduced the umbra Lagrangian-Hamiltonian mechanics approach by utilizing virtual time as umbra time. The approach involved the development of an extended form of the equation of motions as umbra-Lagrange's equation. Some other studies by Mukherjee et al. (2001, 2006, and 2011) further illustrate concepts of umbra Lagrangian. The approach was applied to analyze thermo-mechanical systems (Rastogi et al., 2011), electromechanical system (Mukherjee et al., 2007) and a one-dimensional continuous system (Mukherjee et al., 2009). Kumar et al. (2012) extend the approach to study the dynamics of a solid rotor system with asymmetry in stiffness. Another study by Jain et al. (2018) utilized the approach to understand the dynamic behavior of multi-rotor-system where symmetries are broken in terms of the rotor stiffness.

Different researcher has conducted some studies to analyze the dynamics of the hollow rotor system. Among them, a major contribution comes from Chen et al (2010) who utilized the concept of local equivalent Young's modulus for understanding the dynamic behavior of a 
motor built-in high speed hollow rotating shaft through experimental and numerical study. Some other studies by Chen et al. (2011, 2012, and 2013) enhanced understanding of the influence of the friction on the natural frequency of the hot fit rotor-hollow shaft assembly. Chen and Yang (2010) also performed an experimental study of a hollow shaft with a hot-fitted rotor to determine the optimal value of interference for dynamical stiffness of a shaft fitted with a hot-fit rotor. In another study, Asdaque and Behera (2014) analyzed the dynamic behavior of hollow tapered shaft-rotor to investigate the effects of length and speed on the dynamics of the hollow tapered shaft-rotor system. Further, Guo et al. (2014) theoretically studied an NES-rotor system by developing motion equations for NES rotor as well as TMD rotor dynamical systems by applying Lagrange's equations and determined amplitude of vibration at resonance condition. One study by Cavalini et al. (2017) established the suitability of the composite hollow shaft as a rotor while other study by Y. Yongfeng et al. studied dynamic behavior of hollow-cracked shaft.

The present work is initiated to develop an extension of Lagrangian Hamiltonian mechanics for hollow rotor systems with asymmetries in stiffness and mass by utilizing the approach introduced by Mukherjee et al. (2009, and 2011). The methodology adopted for analysis is presented in sufficient detail for two case studies in subsequent sections.

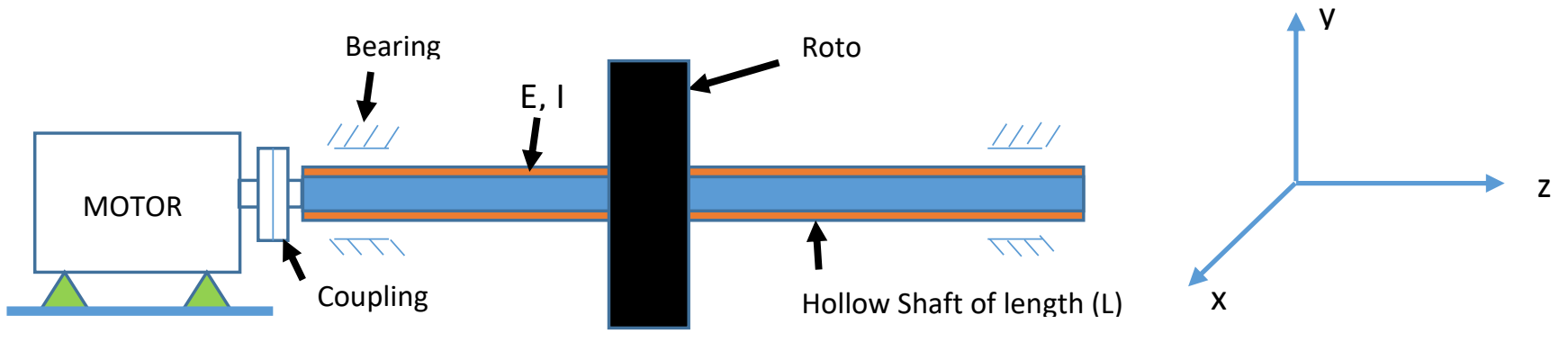

Figure 1 Rotor with DC motor having asymmetries in stiffness

\section{CASE STUDY I: INSTABILITIES DUE TO ASYMMETRY IN STIFFNESS}

This case study considers a rotor with internal and external damping and transverse stiffness due to flexure of the shaft driven by a DC motor as shown in Figure 1. The rotor model admits small asymmetries in stiffness. The work represents the rotor by two discrete pairs of equivalent 
springs and dashpots in the two orthogonal transverse directions. Such dampers include external damping and a part of the internal damping. The study considers the additional circulatory forces due to the internal damping by using modulated gyrator elements in the bond graph model. The analysis neglects shaft twist due to high torsional stiffness assumption.

The umbra-Lagrangian is derived from bond graph model of rotor system by adopting the method developed by Mukherjee $(1997,2001,2011)$ and is expressed as

$$
\begin{aligned}
& L^{*}=\frac{1}{2} m \dot{x}^{2}(\eta)+\frac{1}{2} m \dot{y}^{2}(\eta)-\frac{1}{2} K \dot{x}^{2}(\eta)-\frac{1}{2}(K-\Delta K) \dot{y}^{2}(\eta)-\frac{1}{2} J \dot{\theta}^{2}(\eta)- \\
& \frac{1}{2} R_{i} \dot{\theta}(t)\{x(\eta) y(t)-x(t) y(\eta)\}-\left(R_{a}+R_{i}\right)(x(\eta) \dot{x}(t)+y(\eta) \dot{y}(t))-\left\{R_{i}(x(t) \dot{y}(t)-\right. \\
& \left.y(t) \dot{x}(t))-R_{b}(\dot{\theta}(t))-\Omega\right\} \theta(\eta)
\end{aligned}
$$

Here the symbol represented the standard quantity. Now by applying the extended Noether's equation, Noether's rate equation for asymmetric rotor is written as

$$
\begin{aligned}
& \frac{\mathrm{d}}{\mathrm{dt}}[\mathrm{m}(\dot{\mathrm{x}}(\mathrm{t}) \mathrm{y}(\mathrm{t})-\dot{\mathrm{y}}(\mathrm{t}) \mathrm{x}(\mathrm{t}))-\Delta \mathrm{Ky}(\mathrm{t}) \mathrm{x}(\mathrm{t})]-\dot{\theta}(\mathrm{t}) \mathrm{R}_{\mathrm{i}}\left(\mathrm{x}^{2}(\mathrm{t})+\mathrm{y}^{2}(\mathrm{t})\right)+\left(\mathrm{R}_{\mathrm{a}}+\right. \\
& \left.\mathrm{R}_{\mathrm{i}}\right)(\mathrm{x}(\mathrm{t}) \dot{\mathrm{y}}(\mathrm{t})-\dot{\mathrm{x}}(\mathrm{t}) \mathrm{y}(\mathrm{t}))=0
\end{aligned}
$$

On assuming an orbit of $x(t)=A \cos \omega t$ and $y(t)=A \sin \omega t$, we get

$$
\dot{\theta}(t)=\omega\left(1+\frac{R_{a}}{R_{i}}\right)
$$

Thus, Noether's rate equation becomes

$$
\frac{d}{d t}[m(\dot{x}(t) y(t)-\dot{y}(t) x(t))-\Delta K y(t) x(t)]=0
$$

The above equation consists of symmetric and un-symmetric part. On equating unsymmetric part to zero, one may obtain invariants of motions as

$$
\frac{\mathrm{d}}{\mathrm{dt}}[\mathrm{m}(\dot{\mathrm{x}}(\mathrm{t}) \mathrm{y}(\mathrm{t})-\dot{\mathrm{y}}(\mathrm{t}) \mathrm{x}(\mathrm{t})]=0
$$

Now, the umbra-Hamiltonian of the system is represented as

$$
\begin{aligned}
& \mathrm{H}^{*}=\frac{\mathrm{p}_{\mathrm{x}}^{2}(\eta)}{2 \mathrm{~m}_{\mathrm{xx}}}+\frac{\mathrm{p}_{\mathrm{y}}^{2}(\eta)}{2 \mathrm{~m}_{\mathrm{yy}}}+\frac{1}{2} \mathrm{Kx}^{2}(\eta)+\frac{1}{2}(\mathrm{~K}-\Delta \mathrm{K}) \mathrm{y}^{2}(\eta)+\frac{1}{2 J} \mathrm{p}_{\theta}^{2}(\eta)-\mathrm{R}_{\mathrm{i}} \dot{\theta}(\mathrm{t})\{\mathrm{x}(\eta) \mathrm{y}(\mathrm{t})-\mathrm{x}(\mathrm{t}) \mathrm{y}(\eta)\}+ \\
& \left(\mathrm{R}_{\mathrm{a}}+\mathrm{R}_{\mathrm{i}}\right)(\dot{\mathrm{x}}(\mathrm{t}) \mathrm{x}(\eta)+\dot{\mathrm{y}}(\mathrm{t}) \mathrm{y}(\eta))+\left\{\mathrm{R}_{\mathrm{i}}(\mathrm{x}(\mathrm{t}) \dot{\mathrm{y}}(\mathrm{t})-\mathrm{y}(\mathrm{t}) \dot{\mathrm{x}}(\mathrm{t}))+\mathrm{R}_{\mathrm{b}}(\dot{\theta}(\mathrm{t}))-\Omega\right\} \theta(\eta)
\end{aligned}
$$

On applying the second theorem of umbra-Hamiltonian, $\lim _{\eta \rightarrow t}\left[\frac{d H_{e}^{*}}{d \eta}\right]=0$, we got 
$R_{i} \dot{\theta}(t)[\dot{x}(\eta) y(t)-\dot{y}(t) x(t)]+\left(R_{a}+R_{i}\right)\left(\dot{x}^{2}(t)+\dot{y}^{2}(t)\right)+\left\{R_{i}(x(t) \dot{y}(t)-y(t) \dot{x}(t))+\right.$ $\left.R_{b}(\dot{\theta}(t)-\Omega)\right\} \dot{\theta}(t)=0$

Assuming an orbit $\mathrm{x}(\mathrm{t})=\mathrm{A} \cos \omega \mathrm{t}$, and $\mathrm{y}(\mathrm{t})=\mathrm{A} \sin \omega \mathrm{t}$, we get

$$
-\mathrm{R}_{\mathrm{i}} \dot{\theta}(\mathrm{t}) \mathrm{A}^{2} \omega+\left(\mathrm{R}_{\mathrm{a}}+\mathrm{R}_{\mathrm{i}}\right) \mathrm{A}^{2} \omega^{2}+\left\{\mathrm{R}_{\mathrm{i}} \mathrm{A}^{2} \omega+\mathrm{R}_{\mathrm{b}}(\dot{\theta}(\mathrm{t})-\Omega)\right\} \dot{\theta}(\mathrm{t})=0
$$

On putting part A of equation (8) equal to zero yields

$$
\dot{\theta}(t)=\omega\left(1+\frac{R_{a}}{R_{i}}\right)
$$

and on putting B part of equation (8) equal to zero gives the amplitude of rotor

$$
A=\sqrt{\frac{R_{b}}{R_{i} \omega}\left[\Omega-\omega\left(1+\frac{R_{a}}{R_{i}}\right)\right]}
$$

When we applylim $\underset{\eta \rightarrow t}{ }\left[\frac{d H_{i}^{*}}{d \eta}\right]=0$, we got the following expression

$$
\frac{1}{2}\left\{\left(\frac{p_{x}^{2}(\eta)}{m_{x x}}+\frac{p_{y}^{2}(\eta)}{m_{y y}}\right)+K x^{2}(\eta)+(K-\Delta K) y^{2}(\eta)+\frac{1}{J} p_{\theta}^{2}(\eta)\right\}=0
$$

Now putting $p_{x}(t)=m_{x x} \dot{x}(t), p_{y}(t)=m_{y y} \dot{y}(t)$ and

$$
\begin{array}{r}
p_{\theta}(t)=J \dot{\theta}(t) \text { in Eq. 11, following equation is obtained } \\
\left(m_{x x} \ddot{x}+K x\right) \dot{x}+\left(m_{y y} \ddot{y}+K y-\Delta K y\right) \dot{y}=0
\end{array}
$$

There are two types of frequencies available in Eq. (12). If symmetry part is considered, one may have

$$
\omega_{x x}=\sqrt{\frac{K}{m}}
$$

If un-symmetric part is considered than we have

$$
\omega_{y y}=\sqrt{\frac{K-\Delta K}{m}}
$$

Since these two vectors are perpendicular to each other, we achieve the final natural frequency as

$$
\omega^{2}=\frac{\omega_{x x}^{2}+\omega_{y y}^{2}}{2} \quad \text { or } \quad \omega=\sqrt{\frac{K-\frac{\Delta K}{2}}{m}}
$$




\section{CASE STUDY-II INSTABILITIES DUE TO ASYMMETRY IN MASS}

This case study aims to understand the behavior of the rotor system where the symmetries are broken in terms of variation in mass. The study considered the same rotor system used in the previous case along with small asymmetries in mass in the $\mathrm{Y}$ direction instead of asymmetry in stiffness. All assumptions made in the previous case study also applicable in the present case.

The umbra-Lagrangian function for the system is expressed as

$$
\begin{aligned}
& L^{*}=\frac{1}{2} m \dot{x}^{2}(\eta)+\frac{1}{2}(m+\Delta m) \dot{y}^{2}(\eta)-\frac{1}{2} K \dot{x}^{2}(\eta)-\frac{1}{2} K \dot{y}^{2}(\eta)-\frac{1}{2} J \dot{\theta}^{2}(\eta)- \\
& \frac{1}{2} R_{i} \dot{\theta}(t)\{x(\eta) y(t)-x(t) y(\eta)\}-\left(R_{a}+R_{i}\right)(x(\eta) \dot{x}(t)+y(\eta) \dot{y}(t))-\left\{R_{i}(x(t) \dot{y}(t)-\right. \\
& \left.y(t) \dot{x}(t))-R_{b}(\dot{\theta}(t))-\Omega\right\} \theta(\eta)
\end{aligned}
$$

By following the procedure as explained in previous case study, the relationship to determine threshold frequency and the amplitude of rotor system are derived as given below:

$$
\begin{aligned}
& \dot{\theta}(t)=\omega\left(1+\frac{R_{a}}{R_{i}}\right) \\
& A=\sqrt{\frac{R_{b}}{R_{i} \omega}\left[\Omega-\omega\left(1+\frac{R_{a}}{R_{i}}\right)\right]}
\end{aligned}
$$

Due to asymmetry of mass along y axis, system will have two types of frequencies. One frequency is obtained by considering symmetry part and given below:

$$
\omega_{x}=\sqrt{\frac{K}{m}}
$$

While other frequency is obtained by considering asymmetric part as given below:

$$
\omega_{y}=\sqrt{\frac{K}{\mathrm{~m} \pm \Delta m}}
$$

The final natural frequency can be expressed as

$$
\omega^{2}=\frac{\omega_{x x}^{2}+\omega_{y y}^{2}}{2} \quad \text { or } \quad \omega=\sqrt{\frac{2 K}{m \pm \Delta m}}
$$




\section{RESULTS AND DISCUSSION}

This section presents the results obtained from the analysis of hollow rotor with asymmetries in mass and stiffness by utilizing an extended Lagrangian Hamiltonian mechanics approach. Simulation analysis of the hollow rotor with different asymmetries are performed by adopting bond graph technique for validating the analytical approach. Next sections include the detailed discussion on analytical and simulation results.

\section{Analytical Results}

Analytical results are obtained by adopting the approach discussed in previous section for both cases and discussed separately in subsequent sections. First section presents discussion of results pertaining to hollow rotor with consideration of asymmetry in stiffness while other section discussed the results pertaining to a hollow rotor system with asymmetry in mass.

\section{Asymmetry in stiffness}

By utilizing the equations developed previously, natural frequency and amplitude of vibration induced in the hollow shaft rotor system are obtained and presented graphically in figure (2-4). The study considers the hollowness ratio as the ratio of the internal diameter to the external diameter. The study calculates these results by assuming excitation frequency as $22 \mathrm{rad} / \mathrm{s}$. The careful analysis of these results reveals an increasing trend in the amplitude of the vibration along with a decreasing trend in the natural frequency of the vibration for a hollow shaft rotor with different hollowness ratios. The results also show that there is no appreciable variation in the performance of the hollow rotor with hollowness ratio up to 0.4 . This shows that hollow shaft with hollowness ratio value more than 0.4 is not suitable to employ for the rotor shaft. Further results are obtained by varying excitation frequencies as $26,30,34$, and $38 \mathrm{rad} / \mathrm{s}$, respectively and no effect observed on natural frequency as expected while increasing trend observed in amplitude of the vibration with increasing value of the excitation frequency as shown in figure 10 (b) for a hollow shaft rotors with the hollowness ratio as 0.2 . Similar trends. 
are observed for hollow shaft rotor with different hollowness ratios. These results are not included here to avoid repeatability.
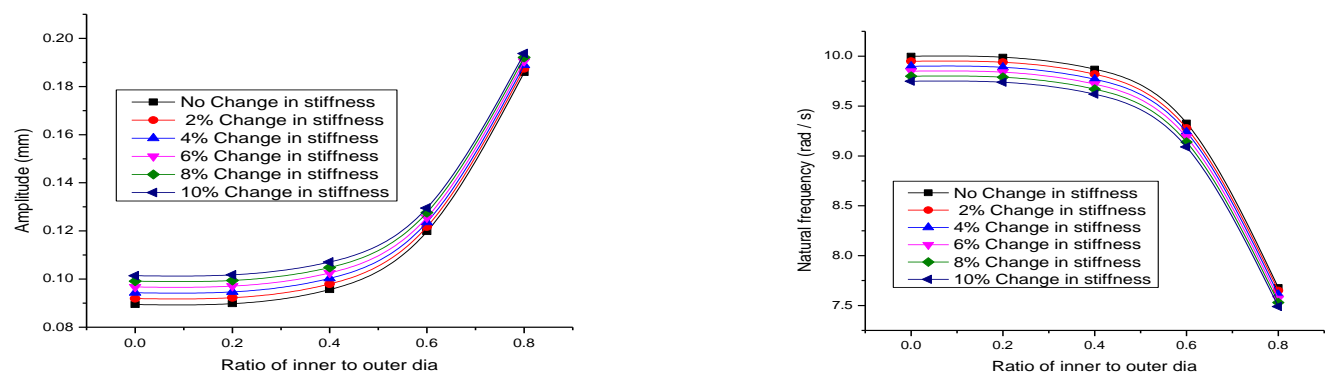

Figure 2 Effect of hollowness on amplitude Figure 3 Effect of hollowness on natural frequency
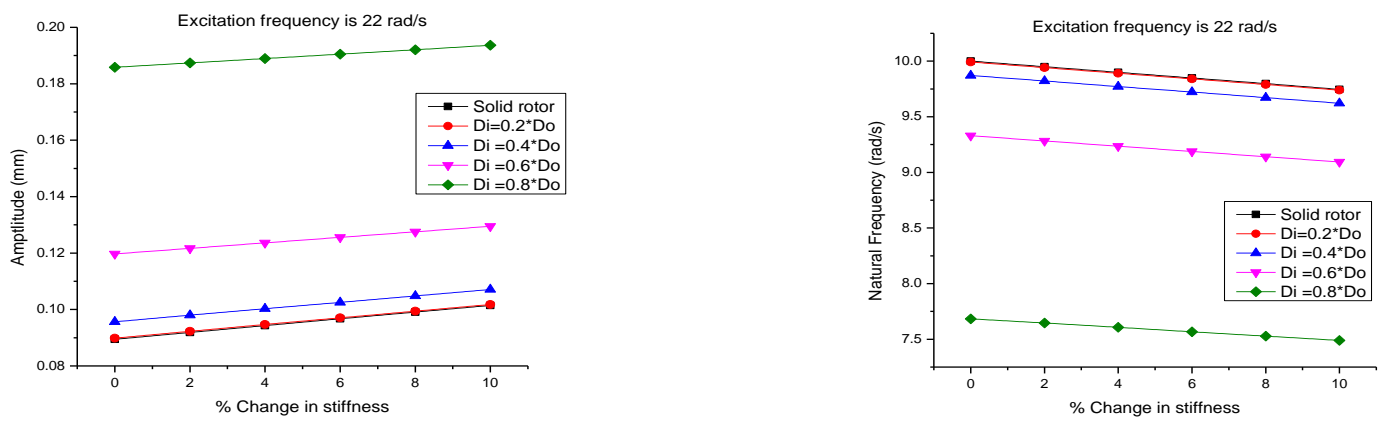

Figure 4 Amplitude Vs \% Change in stiffness

Figure 5 Natural frequency Vs \% Change in stiffness

\section{Asymmetry in mass}

By utilizing, the equations developed in previous section, the amplitudes and the natural frequencies of the vibration for a hollow rotor system having varying percentages of mass asymmetries along the y-axis obtained and presented in Figure 6 to Figure 9. The results depict the decreasing trend in the natural frequency of the system with increasing percentage of asymmetries of mass along the $y$-axis for hollow rotor with different hollowness ratios while reverse trend observed in the amplitudes of the vibrations with an increasing percentage of mass asymmetry. The results also show that there is little variation in the performance of a hollow shaft in comparison with a solid shaft if the hollowness ratio of the hollow shaft rotor is kept 
below a certain limit (i.e. 0.4 ). The results also obtained by varying the excitation frequency and observed no affect in the excitation frequency while amplitudes increases with an increase in excitation frequency as shown by the figure 10 (a).

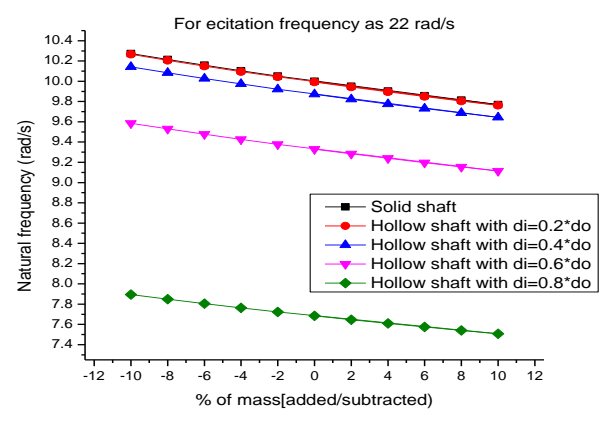

Figure 6 Frequency Vs \% Change in mass

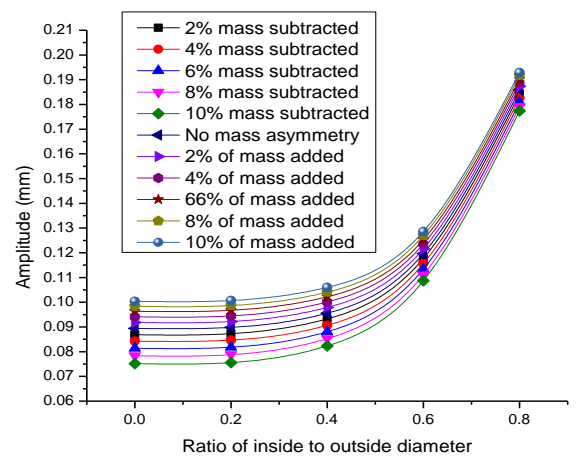

Figure 8 Effect of hollowness on amplitude

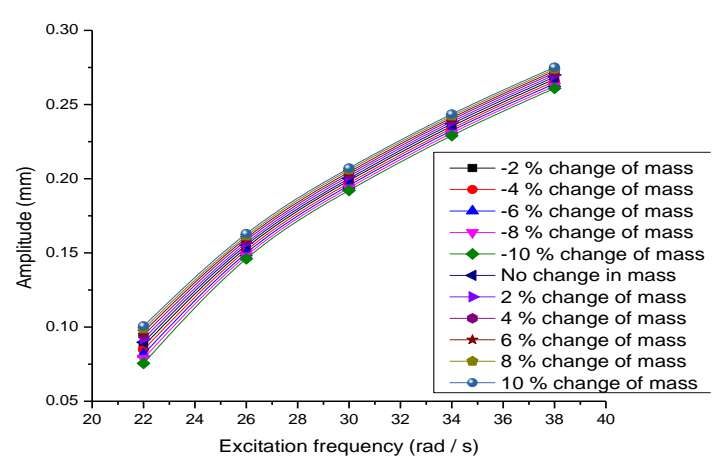

(a) Hollow rotor with asymmetry in mass

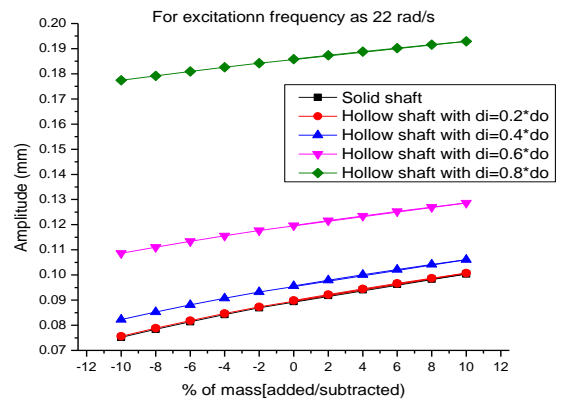

Figure 7 Amplitude Vs \% Change in mass

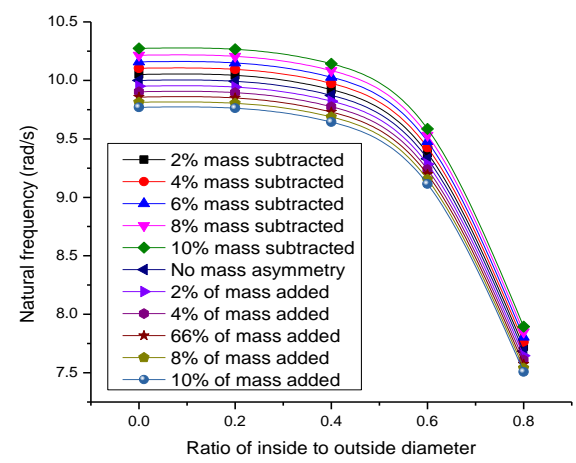

Figure 9 Effect of hollowness on natural frequency

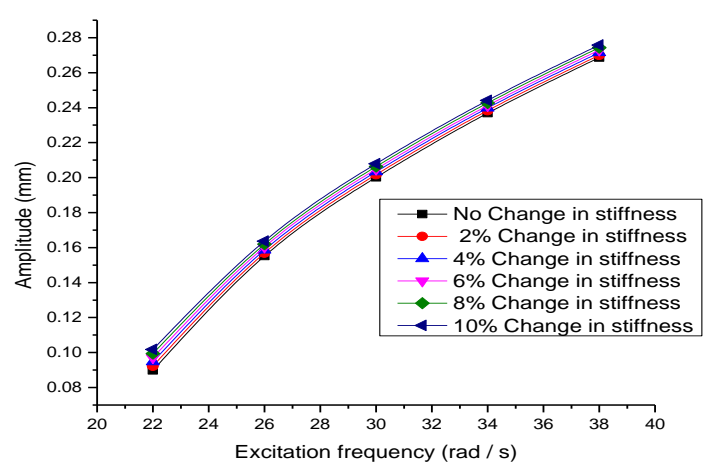

(b) Hollow rotor with asymmetry in stiffness Figure 10 Effect of Excitation frequency on amplitude of hollo rotor when $D_{i}=0.2 * D_{o}$ 


\section{Simulation Results}

The main purpose of this study is to gain an insight into dynamical behavior. Moreover, it provides validation of the theoretical results obtained in the previous section. The bond graph model of an asymmetric rotor with internal and external damping was developed and simulated through the software SYMBOLS-Shakti. The study performs a series of experimentation to show the effectiveness of the analysis described above. The work includes the simulation study performed for both the cases discussed in the previous section and presented in succeeding subsections. For simulation analysis of the hollow rotor with small asymmetry in stiffness and mass, the present work considers different input parameters as shown in Table-1. Amplitudes and threshold frequencies of the vibratory behavior of the hollow rotor with asymmetries are determined through the bond graph technique and presented graphically along with the corresponding analytical results through figures 14 to 17 . Figures 14 and 15 presents the results for a hollow rotor with asymmetry in stiffness while figures 16 and 17 represent the corresponding results for a hollow rotor with asymmetry in mass. The threshold frequency of the hollow rotor system is found to be twice of the natural frequency of the hollow rotor system. The simulation results are in harmony with the analytical results and thus proves the utility of the extended Lagrangian Hamiltonian approach for predicting the dynamic behavior of hollow rotor with small asymmetries.

Table 1. Simulation parameters for hollow rotor with asymmetry in stiffness

\begin{tabular}{|c|c|c|c|c|c|c|c|}
\hline Parameter & $\mathrm{K}$ & $\mathrm{m}$ & $\mathrm{J}$ & $\mathrm{R}_{\mathrm{i}}$ & $\mathrm{R}_{\mathrm{a}}$ & $\mathrm{R}_{\mathrm{b}}$ & $\Omega$ \\
& $(\mathrm{N} / \mathrm{m})$ & $(\mathrm{Kg})$ & $\left(\mathrm{Kg}-\mathrm{m}^{2}\right)$ & $(\mathrm{N}-\mathrm{s} / \mathrm{m})$ & $(\mathrm{N}-\mathrm{s} / \mathrm{m})$ & $(\mathrm{N}-\mathrm{s} / \mathrm{m})$ & $(\mathrm{rad} / \mathrm{s})$ \\
\hline Value & 1000 & 10 & 1 & 5 & 5 & 0.2 & 22 \\
\hline
\end{tabular}



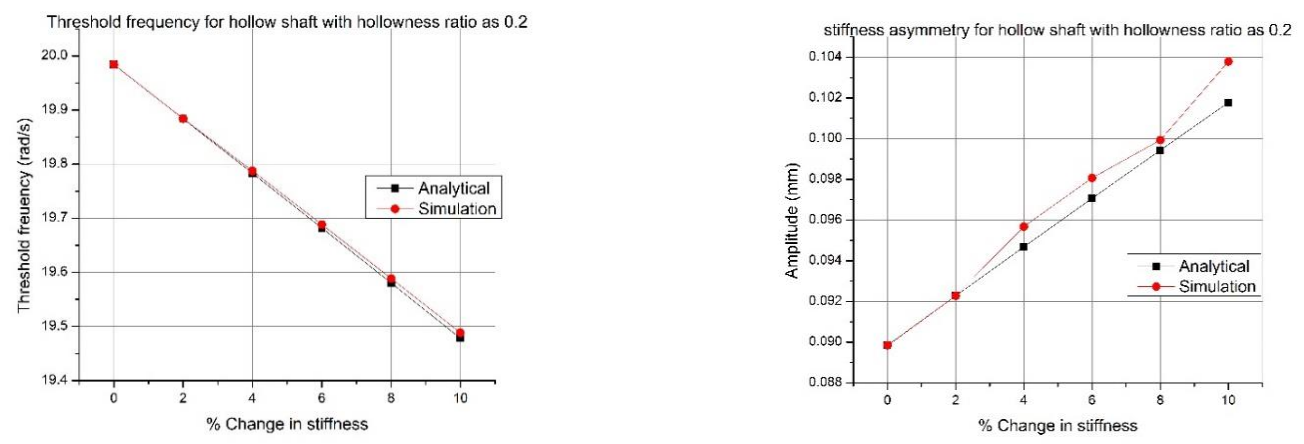

(a) Effect on threshold frequency

(b) Effect on amplitude

Figure 11 Effect of stiffness asymmetry on hollow rotor system

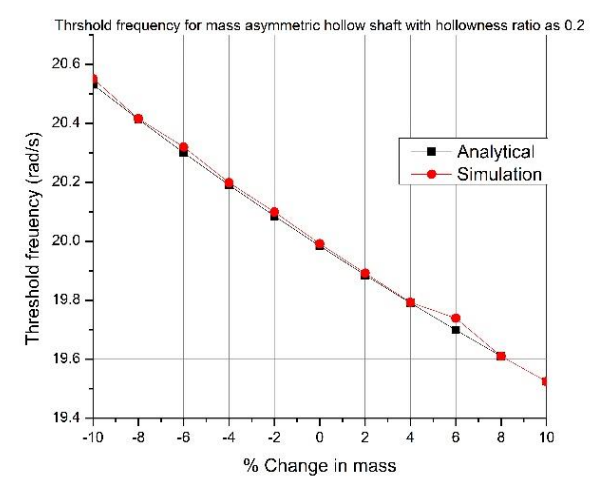

(a) Effect on threshold frequency

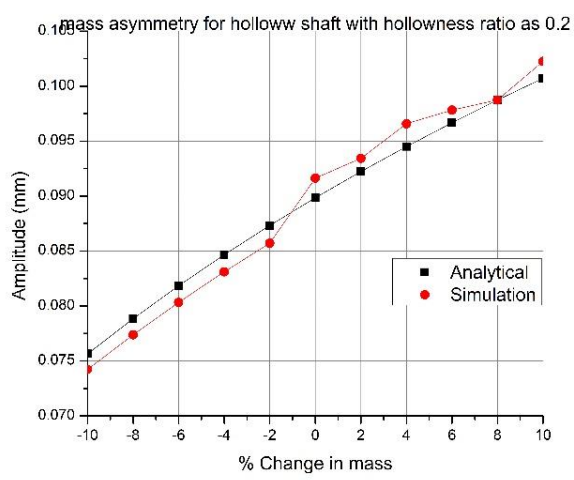

(b) Effect on amplitude

Figure 12 Effect of stiffness asymmetry on hollow rotor system

\section{CONCLUSION}

This work presents an analytical framework for understanding the changes in the dynamic behavior of the hollow rotor system caused by the asymmetries in terms of stiffness and mass of the shaft rotor. Following conclusion has been drawn from the study:

- The study develops expressions for determining the natural frequency and the amplitude of vibration of a hollow shaft rotor through Extended Lagrangian Hamiltonian formalism.

- The study obtains the umbra-Lagrangian function from its bond graph model, which remains invariant and leads to significant aspects of limiting dynamics when used with umbra Hamiltonian. The results exhibits marginal difference in the 
behavior of a hollow rotor with a hollowness ratio less than 0.4 in comparison to a solid rotor. This fact justify the suitability of a hollow rotor for industrial purpose.

- The results of simulation study are in harmony with the analytical results and thus validate the limiting behavior obtained through present study.

- Harmony of simulation results with analytical results justify the use of an extended Lagrangian Hamiltonian approach for predicting the dynamic behavior of hollow rotor with small asymmetries.

- Further present study can be extended to incorporate more asymmetries in the hollow rotor like as transverse crack, circular crack and multi rotor system.

\section{REFERENCES}

Dimarogonas, 1996. Vibration of cracked structure: a state of the art review, Eng. Fract. Mech. $55831-857$.

C. Kumar, V. Rastogi, 2009. A brief review on dynamics of a cracked rotor, Int. J. Rot. Mach. 1-6, https://doi.org/10.1155/2009/758108. Article ID 758108.

B. Vujanovic, 1978. Conservation laws of dynamical systems via d'Alembert's principle, Int. J. Nonlinear Mech. 13 185-197.

B. Vujanovic, S.E. Jones, 1989. Variational Methods in Nonconservative Phenomena, Academic press, Boston

D. S. Djukic, 1973. A procedure for finding first integrals of mechanical systems with gaugevariant Lagrangians, Int. J. Nonlinear Mech. 8 479-488.

D. S. Djukic, B. Vujanovic, 1975. Noether's theory in classical nonconservative mechanics, Acta Mech. 23 17-27.

D. C. Karnopp, 1977. Lagrange's equations for complex bond graph systems, J. Dyn. Sys. Meas. Control 99 300-306. 
A. Mukherjee, A.K. Samantaray, 1997. Umbra-Lagrange's equations through bond graphs, in: Proc. of ICBGM - International Conference on Bond Graph Modeling and Simulation, Phoenix, Arizona, USA, pp. 168-174.

E. Noether, 1971 Invariant variation problems, Transp. Theory Stat. Phys. 1 186-207.

A. Mukherjee, 1994. Junction structures of bond graph theory from analytical mechanics viewpoint, in: Proc. of CISS-1st Conference of International Simulation Societies. Zurich, Switzerland, pp. 661-666.

A. Mukherjee, V. Rastogi, 2006. A. Das Gupta, A methodology for finding invariants of motion for asymmetric systems with gauge-transformed Umbra lagrangian generated by bond graphs, Simulation 82 207-226.

A. Mukherjee, V. Rastogi, A. Dasgupta, 2011. Revisiting Umbra-Lagrangian-Hamiltonian mechanics: its variational foundation and extension of Noether's theorem and poincare-Cartan Integral, Int. J. Nonlinear Mech. 46 745-757.

V. Rastogi, A. Mukherjee, 2011. Conservation laws for gauge-variant Umbra-Lagrangian in Classical mechanics using Bond graphs, Simulation 87 301-312.

A. Mukherjee, V. Rastogi, A. Dasgupta, 2009. Extension of Lagrangian-Hamiltonian mechanics for continuous systems e investigation of dynamics of a one dimensional internally damped rotor driven through a dissipative coupling, Nonlinear Dyn. 58 107-127.

C. Kumar and Vikas Rastogi, 2012. Effects of asymmetric stiffness on parametric instabilities of rotor, Journal of computation and applied research in mechanical engineering, Vol 1 No 2, 119-128.

Anuj Kumar Jain , Vikas Rastogi , Atul Kumar Agrawal, 2018. A novel approach to study effects of asymmetric stiffness on parametric instabilities of multi-rotor-system, Journal of Sound and Vibration 413 159-172.

S. Y. Chen, C. Kung, T. T. Liao, \& Y. H. Chen, 2010. Dynamic effects of the interference 
fit of motor rotor on the stiffness of a high-speed rotating shaft, Transactions of the Canadian Society for Mechanical Engineering, 34(2), 243-261.

S. Y. Chen, C. Kung, Jung-Chun Hsu, 2011. Dynamic Analysis Of A Rotary Hollow Shaft With Hot-Fit Part Using Contact Elements With Friction, Transactions of the Canadian Society for Mechanical Engineering, Vol. 35, No. 3.

Shin-Yong Chen, 2013. The Dynamic Analyses and Verifications of A Hollow shaft With HotFit Component Using 3d Finite Contact Element, Transactions of the Canadian Society for Mechanical Engineering, Vol. 37, No. 1.

S. Y. Chen, 2012 . An equivalent direct modeling of a rotary shaft with hot-fit components using contact element modal analysis results, Computers and Mathematics with Applications, 64(5), 1093-1099.

Shin-Yong Chen, Shyi-Kae Yang, 2010. The Optimal study of Finite Element Modelling for a High Speed Hollow Shaft with a Hot-fitted Motor Rotor, Internal symposium on Computer, Communication and Automation.

P M G Bashir Asdaque, R K Behera, 2014. Vibration Analysis of Hollow Profiled Shafts", International Journal of Current Engineering and Technology, Special Issue-3, 124-128.

C Guo, MA AL-Shudeifat, AF Vakakis, LA Bergman, DM McFarland, J Yan, 2014. “A. Vibration reduction in unbalanced hollow rotor systems with nonlinear energy sinks", Nonlinear Dynamics 79 (1), 527-538

Aldemir Ap Cavalini Jr, Thiago A M Guimarães, Bruno R M G da Silva, Valder Steffen Jr 2017. "Analysis of the Dynamic Behavior of a Rotating Composite Hollow Shaft", Latin American Journal of Solids and Structures 14, 1-16.

Yongfeng Yang, Qinyu Wu,, Yanlin Wang, Weiyang Qin, Kuan Lu, 2019 "Dynamic characteristics of cracked uncertain hollow-shaft”, Mechanical Systems and Signal Processing $124,36-48$ 